\title{
Cardiac hypertrophy and heart failure: From the case to review of literature
}

\author{
Francesco Massoni ${ }^{*}$, Lidia Ricci, Claudio Simeone, Emanuela Onofri, Serafino Ricci \\ Departments of Anatomical Sciences, Histological, Legal Medicine, and Locomotor Apparatus, Sapienza University of \\ Rome, Rome, Italy
}

\begin{abstract}
In response to an increased workload due to physiological or pathological stimuli, the heart may undergo a process of growth with increased muscle mass called cardiac hypertrophy. It is a particular mechanism of long term compensation used by the heart to adapt permanently to a greater workload.

Although, through its peculiar structural, molecular and metabolic characteristics, in early stage the hypertrophy allows to maintain an adequate cardiac function, after a variable period of time, the same characteristics promote the evolution to contractile dysfunction and heart failure. The latter represents an important cause of death and so the cardiac hypertrophy increases the cardiovascular morbidity and mortality.

In this paper we report a rare case of extremely high degree of concentric cardiac hypertrophy, with a heart weight of 1050 $\mathrm{g}$ and longitudinal diameter of $16.5 \mathrm{~cm}$, transverse diameter of $16 \mathrm{~cm}$ and antero-posterior diameter of $9 \mathrm{~cm}$. The thickness of the left ventricle free wall was $4.2 \mathrm{~cm}$, of the septum $4.3 \mathrm{~cm}$ and at the apex level $3.5 \mathrm{~cm}$.

These data, compared with those described in scientific literature, indicate the exceptional nature of our necropsy finding of a huge cardiac hypertrophy.

The analysis of the pathogenetic mechanisms, which may determinate the fatal event in case of cardiac hypertrophy, shows that in the described case the death cause can be the onset of heart failure in presence of cardiomegaly.
\end{abstract}

Key Words: Cardiac hypertrophy, cardiomegaly, heart failure, heart weight

\section{Introduction}

In response to an increased workload due to physiological or pathological stimuli, the heart may undergo a process of growth with increased muscle mass, the cardiac hypertrophy, in which the increase in weight is greater than $95 \%$ of the normal limit in relation to body weight and sex (1).

The estimated prevalence of left ventricular hypertrophy in the population is around 16\% (2).

Cardiac hypertrophy is a unique mechanism of long term compensation used by the heart to adapt permanently to a greater workload. This may be due to a pressure overload (like in hypertensive patients and in people who practice power sports, such as weight lifting) or to a volume overload (like in endurance sports such as cycling, marathon, swimming or in the case of heart valve insufficiency) (3).

Although representing a compensation mechanism, several studies showed that cardiac hypertrophy increases the cardiovascular morbidity and mortality $(4,5)$. In early stage, it can maintain adequate cardiac function, but after a variable period of time may evolve to contractile dysfunction and heart failure (3). In fact, the peculiar and unusual structural, molecular and metabolic features of hypertrophic heart, that in the initial phase allow an increase of cardiac function, over a certain limit decrease the contractile efficiency and cause the evolution of hypertrophy in heart failure (6). The latter represents an important cause of death, presenting an annual mortality rate ranging between $10 \%$ and $20 \%$ depending on its severity, reaching $30-40 \%$ at one year after the onset of symptoms (7).

In this paper we report a rare case of extremely high degree of concentric cardiac hypertrophy (cardiomegaly) and a detailed analysis of the pathogenic mechanisms occurring in the determination of deadly event.

\section{Case report}

The case involved a 45 year old woman, in apparent good health. The body, with a length of about $170 \mathrm{~cm}$ and weighing about $90 \mathrm{~kg}$ $(\mathrm{BMI}=31.14)$, was in good general hygienic conditions. The body showed no signs of harmfulness and toxicological control was negative for common drugs of abuse. 
The examination of the thanatological phenomena showed purple hypostasis in lower parts of the back, rising to the anterior axillary, and rigor mortis was present and more valid in the upper half of the body than in the bottom.

To the opening we found the integrity of the diaphragm and bowel loops and the absence of fluid in the abdominal cavity. Following removal of the sternal plastron, at the level of the sternal head of the II-III rib, a non infiltrated transversal fracture was detected, as probable outcome of external cardiac massage, and pleural cavity was free from adhesions and dry. Pericardial cavity was free from adhesions with the presence of a few cc of serous fluid.

The heart was significantly increased in shape and volume, with a weight of $1050 \mathrm{~g}$ and longitudinal diameter (LD) of $16.5 \mathrm{~cm}$, transverse diameter (TD) of $16 \mathrm{~cm}$ and antero-posterior diameter (APD) of $9 \mathrm{~cm}$ (Figure 1), and on palpation the consistency was significantly increased.

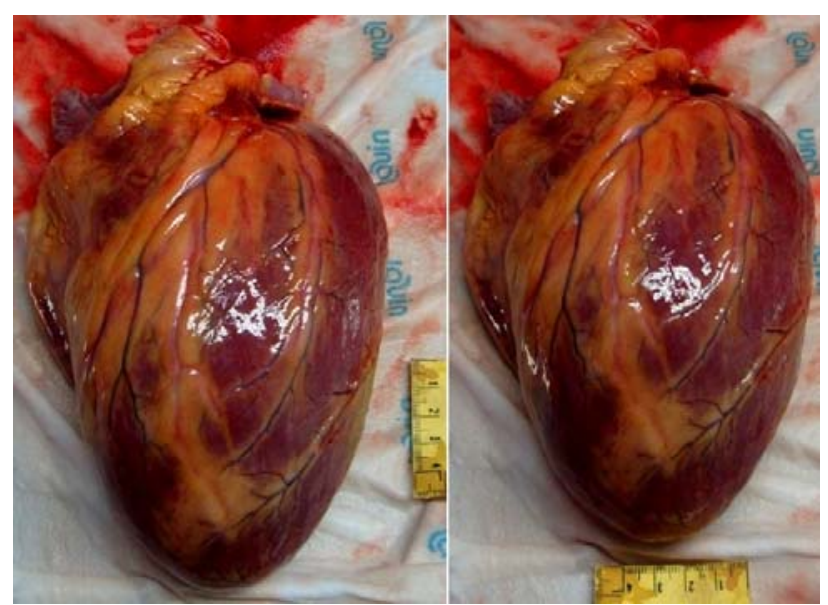

Fig. 1. Image of the heart: note the excessive size of diameters.

The heart apex showed an important left ventricular hypertrophy, confirmed by the section of the rest of the organ, with a thickness of the left ventricle free wall of $4.2 \mathrm{~cm}$, of the septum of $4.3 \mathrm{~cm}$ and at the apex level of $3.5 \mathrm{~cm}$, and a pale myocardium (boiled flesh-colored) (Figure 2).

The papillary muscles were hypertrophic and chordae tendineae were slightly thickened. The valvular systems showed no alterations. To the opening of the coronary arteries according to cross sections of $1 \mathrm{~cm}$, the walls were dilated with patent lumen.

The lungs were increased in shape, volume and consistency $(830 \mathrm{~g}$ the right and $800 \mathrm{~g}$ the left), with some petechiae in the interlobar fissures and sub crackling to palpation. Large, medium and small bronchi showed patent lumen and hyperemic mucosa and at the opening of vessels leakage of fluid blood. The full thickness section of the organs showed red and spongy cut surface, with frankly congested and edematous appearance, finding corroborated by the squeezing of the viscera with leakage of copious amounts of serum hematic fluid, partially frothy.

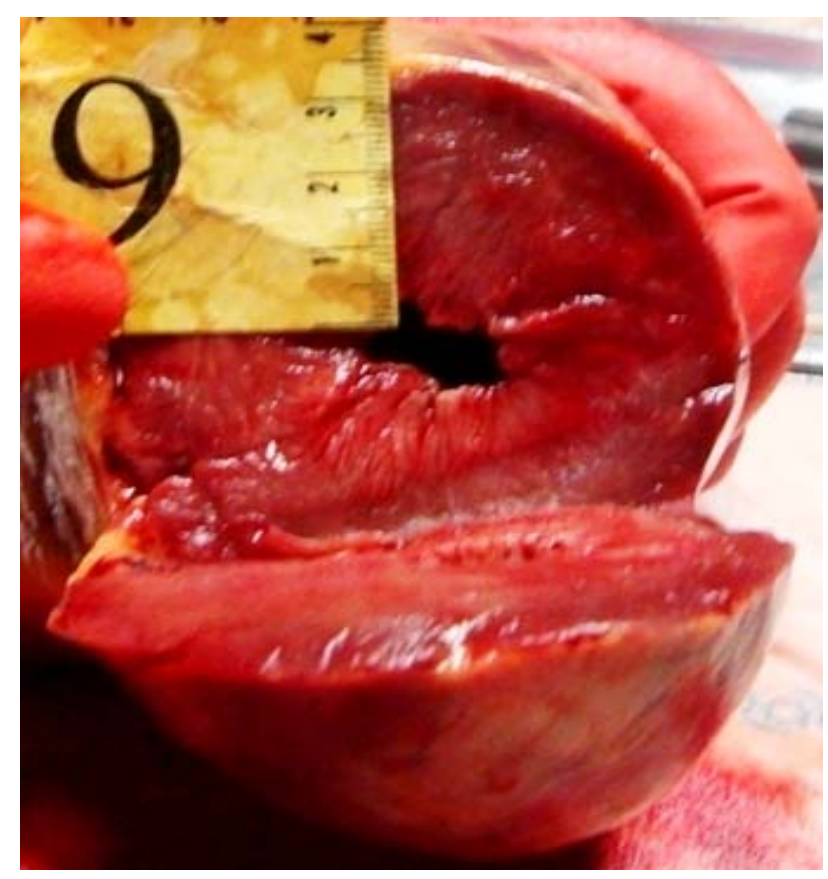

Fig. 2. Section at heart apex: note the significant thickening of ventricular walls with decrease in cavity radius.

\section{Discussion}

In literature the hypertrophic heart weights found in case of autopsy are variable. In a study on patients affected by hypertrophic cardiomyopathy, a genetic form of hypertrophy, the average heart weight was $530.0 \pm 225.1 \mathrm{~g}$ and the average thickness of the interventricular septum was 2.25 $\pm 0.53 \mathrm{~cm}$ (8), while in a study on deceased obese patients the weight ranged between $450 \mathrm{~g}$ and 820 $\mathrm{g}$ (average weight $619 \mathrm{~g}$ ) and the wall thickness of the left ventricle between 1.5 and $3.0 \mathrm{~cm}$ (average thickness $2.0 \mathrm{~cm}$ ) (9). In recent literature cases the heart weight ranged between $405 \mathrm{~g}$ (10) and $560 \mathrm{~g}$ (11).

The severity of pathological hypertrophy and the heart weight (normal values: 250-300 $\mathrm{g}$ in women and $300-350 \mathrm{~g}$ in men, approximately between $0.4 \%$ and $0.5 \%$ of body weight) depend on the basic pathological condition (usually $600 \mathrm{~g}$ in pulmonary hypertension and $800 \mathrm{~g}$ in systemic hypertension, aortic stenosis and mitral

East J Med Volume:21, Number:4, October-December/2016 
regurgitation) (12). In rare cases of extreme pathological conditions (aortic regurgitation and hypertrophic cardiomyopathy) was found an exceptional increase in heart weight up to $1000 \mathrm{~g}$ and an increase in the diameter of individual muscle fibers (from $18 \mu$ to $27 \mu$ ) (13).

These data show that our case is an exceptional necropsy finding of cardiac hypertrophy, because the heart weight is $1050 \mathrm{~g}$ and the left ventricular walls thicknesses ranges between 3.5 and $4.2 \mathrm{~cm}$, compared to values of the normal which ranges between 1.3 and $1.5 \mathrm{~cm}$ (12).

Morphologically (shape of the heart) and in relation to the stimulus which supports the development, cardiac hypertrophy is distinguished in concentric and eccentric. In this case the macroscopic heart observation indicated the presence of a pathological left ventricular concentric hypertrophy. This anatomopathological feature is characterized by an increase in ventricular wall thickness (with reduction in the capacity of ventricular distention) and by the reduction of ventricular cavity radius (small ventricular cavity), with a consequent raise in the ratio between the two parameters, and individual cardiomyocytes show a greater enhancement in width compared to the length, with decrease in the length/width ratio (14). The thickness growth reduces stress on ventricular walls, allowing the heart to maintain or even increase the function of pump, according to Laplace law (15), whereby wall stress (or tension) is an inverse function of the wall thickness $[$ tension $=($ pressure $\times$ radius $) /(2 \times$ thickness $)]$.

The pathological concentric hypertrophy is due to a chronic pressure overload, as occurs in hypertension or in cases of aortic and pulmonary valve stenosis (16). These pathological stimuli cause ventricular remodeling, dilatation and fibrosis, which result in the onset of the most frequent pathological cardiac hypertrophy (17), which differs from the physiological form for the specific structural and molecular characteristics.

The pathologic cardiac hypertrophy, unlike the physiological, is only partially reversible upon cessation of the causing stimulus and after a variable period of time may become inadequate to maintain an efficient cardiac function and evolves in heart failure with an increased mortality (16). In fact, although both forms are characterized by cardiomyocytes enlargement, during pathological hypertrophy a modification of interstitial tissue, occurs with increase in the number of fibroblasts that synthesize excessive amounts of extracellular matrix proteins, particularly collagen, causing a marked interstitial fibrosis that does not occur in physiological form (18). This results in an increase in the rigidity of the walls, contributing to the onset of a initial diastolic and then systolic dysfunction in the hypertrophic heart. Previous alteration is associated with the growth of capillary network, which in pathological hypertrophy is not proportionate to the increase in myocardial mass (19). Even at the molecular level, studies have established the existence of differences between physiological and pathological hypertrophy. Unlike the latter, in pathological hypertrophy there is a stress-induced reactivation of the genes that encode the fetal isoforms of proteins, normally produced during the development process of the cardiac cells (20). The reactivation of fetal genetic program induces the production of larger amounts of some proteins, including the Atrial Natriuretic Peptide (ANP), B-type natriuretic peptide (BNP) and the fetal isoforms of contractile proteins $(\alpha$-actin and $\beta$-myosin heavy chain, $\beta-\mathrm{MHC}$ ), and decreased or altered production of adult proteins $(\alpha$-myosin heavy chain protein and sarcoplasmic reticulum $\mathrm{Ca} 2+-$ ATPase) (16). Synthesis of contractile protein's fetal isoforms allows a greater energy saving at the expense of reduced functionality, mainly due to lower consumption of ATP by the myosin's fetal isoform.

Finally at the metabolic level in the pathological hypertrophy there is a change of the metabolic substrates for the production of energy in the cardiomyocytes. During heart development, glycolysis is the major source of energy for muscle cells, while after birth the major metabolic pathway is the fatty acid oxidation, due to increased activity of mitochondria (21). In the pathological hypertrophic heart there is a return to a prevalent glycolytic metabolic profile (22).

In the described case, the subject's excessive weight (BMI=31.14) could lead one to suppose, as demonstrated by scientific evidence (23), the existence of hypertension that, by requiring a work overload, causes a left ventricular pathological hypertrophy, which is a risk factor for heart failure. Furthermore it was shown that the cardiomegaly is associated with the only presence of obesity indicators in the absence of arterial hypertension (24) and that it can be the leading factor of death in obese subjects.

An anatomopathological feature of huge left ventricular hypertrophy, as the one described, implies a particularly high risk of progression to heart failure, compared to ones in which the degree of hypertrophy is lower (25). In fact over a

East J Med Volume:21, Number:4, October-December/2016 
certain limit, the structural, molecular and metabolic abnormal characteristics of cardiac hypertrophy influence heart's contractile efficiency and causing progression to failure.

The first hypothesized mechanism causing this evolution has been the loss of cardiomyocytes as a result of ischemic necrosis. This is primarily due to the decrease of the surface/volume ratio which causes a lower diffusion of metabolic substrates, especially oxygen, into the hypertrophic cells, influencing a low supply to the mitochondria in the center of cardiomyocytes with the consequent deficient energy production in the form of ATP, which, itself, results in depressed contractile efficiency (26). The insufficient development of the capillary network and the increased intercapillary distance contribute to insufficient supply of oxygen to the heart muscle cells. In fact the capillary growth does not proceed in proportion to the cardiomyocytes hypertrophy, causing the decrease of the oxygen diffusion through the myocardium (27). In addition, interstitial fibrosis of cardiac hypertrophy creates a barrier to the diffusion of metabolic substrates and oxygen to the muscle cells, contributing to ischemic necrosis and myocardial contractile insufficiency.

Experimental observations have shown that autophagy can contribute to the loss of contractile myocardial cells. This process physiologically provides the renewal of cellular proteins, but in the hypertrophic cardiomyocytes proceeds at a high rate, leading to cell death (28).

Some studies $(29,30)$ suggest that cardiomyocyte apoptosis can participate to the transition from compensated hypertrophic phase to the onset of myocardial contractile deficit till to heart failure and death, through the loss of cardiac cells and their replacement by fibrous tissue. It is assumed that the continuous stimulation of cell growth induced by chronic work overload and the reactivation of the expression of fetal genes may induce an abortive response in the heart muscle cells and their apoptosis, because they are definitively differentiated and unable to progress in cell cycle up to the mitotic division (31).

The loss of cells is accompanied by the onset of a pronounced interstitial fibrosis, due to the quantitative and qualitative abnormal accumulation of collagen, which determines a lower distensibility and an increased stiffness of the heart walls, leading to diastolic dysfunction that contributes to heart failure $(16,18,32)$.
The excessive heart weight, the pale myocardium (boiled flesh-colored) and greatly increased consistency show that the changes listed are present in our case, conditioning the contractile efficiency of the heart.

In addition to previous mechanisms, the hypertrophic heart dysfunction is due to the change of the cardiomyocytes contractile characteristics, caused by the re-expression of fetal contractile proteins (eg $\beta$-MHC) and of the proteins regulating intracellular calcium homeostasis (sarcoplasmic reticulum $\mathrm{Ca}^{2+}$ ATPase). The fetal isoforms of contractile proteins have a reduced efficiency in using ATP during contraction and this unfavorable bioenergetic profile plays an important role in hypertrophic heart dysfunction and in its evolution to heart failure (33). The sarcoplasmic reticulum $\mathrm{Ca} 2+-$ ATPase play the fundamental function of restoring the normal cytosolic calcium concentration after the contraction, promoting the relaxation of actin and myosin filaments. During hypertrophic process, the decreased levels of production and activity of the $\mathrm{Ca} 2+$-ATPase, due to abnormal gene expression, lead a slow removal of calcium ions and a compromise restore of their intracellular concentration to diastolic levels, causing a deficient diastolic relaxation and the onset of heart failure (34).

Finally, because the heart is the organ which uses the greatest amount of energy ( $6 \mathrm{~kg}$ of ATP/day), it needs a constant supply to perform its contractile function. In course of cardiac hypertrophy in the mitochondria there is a switch from the fatty acid oxidation to a predominantly glycolytic metabolism. Due to the smaller amount of ATP per gram supplied by carbohydrates than to fatty acids, the switch causes an insufficient energy production to the needs of hypertrophic heart and makes the contraction process of the heart muscle cells less efficient, influencing the transition from compensate to decompensate hypertrophy (35). The lethal consequences can be myocardial infarction (36), rupture (37), haemopericardium (38) or arrhythmia (39) and their early diagnosis is very important.

Cardiac hypertrophy is a compensatory mechanism of many diseases which cause a chronic work overload in the heart muscle, but at the same time is an important risk factor for death in patients who are affected.

Although the occurrence of cardiac hypertrophy is quite frequent at the autopsy, in literature heart 
weights are lower than in our case, whose death can be attributed to the onset of heart failure in presence of a huge cardiomegaly.

\section{References}

1. Chugh SS, Kelly KL, Titus JL. Sudden cardiac death with apparently normal heart. Circulation 2000; 102: 649-654.

2. Gosse P, Dallocchio M. Left ventricular hypertrophy: epidemiological prognosis and associated critical factors. Eur Heart J 1993; 14: 16-21.

3. McMullen JR, Jennings GL. Differences between pathological and physiological cardiac hypertrophy: novel therapeutic strategies to treat heart failure. Clin Exp Pharmacol Physiol 2007; 34: 255-262.

4. Bella JN, Göring HHH. Genetic epidemiology of left ventricular hypertrophy. Am J Cardiovasc Dis 2012; 2: 267-278.

5. Vakili BA, Okin PM, Devereux RB. Prognostic implications of left ventricular hypertrophy. Am Heart J 2001; 141: 334-341.

6. Katz AM. Maladaptive growth in the failing heart: the cardiomyopathy of overload. Cardiovasc Drugs Ther 2002; 16: 245-249.

7. McMurray JJ, Pfeffer MA. Heart failure. Lancet 2005; 365: 1877-1889.

8. Morimoto S, Sekiguchi M, Hiramitsu S, et al. Contribution of cardiac muscle cell disorganization to the clinical features of hypertrophic cardiomyopathy. Heart Vessels 2000; 15: 149-158.

9. Cummings PM, Le BH, Lopes MBS. Postmortem findings in morbidly obese individuals dying after gastric bypass procedures. Hum Pathol 2007; 38: 593-597.

10. Di Paolo M, Guidi B, Bugelli V, Naccarato GA. Sudden death of a sportsman. An unusual case of idiopathic form of pulmonary hypertension. Int J Legal Med 2012; 126: 52.

11. Giraldo RD, Guarin CV, Viloria VB. Sudden death: intramural coronary artery. Int J Legal Med 2012; 126: 196.

12. Schoen FJ, Mitchell RN. The Heart. In: Kumar V, Abbas AK, Fausto N, Aster J. Robbins and Cotran Pathologic Basis of Disease - 8th ed. Philadelphia: Saunders Elsevier; 2010, p. 529-587.

13. Hort W. Myocardial hypertrophy. Light microscopic findings on the myocardium. Blood supply. Ventricular dilatation and heart failure. Basic Res Cardiol 1977; 72: 203-208.

14. Dorn GW. The fuzzy logic of physiological cardiac hypertrophy. Hypertension 2007; 49: 962 970 .
15. Norton JM. Toward consistent definitions for preload and afterload. Adv Physiol Educ 2001; 25: 53-61.

16. Bernardo BC, Weeks KL, Pretorius L, McMullen JR. Molecular distinction between physiological and pathological cardiac hypertrophy: experimental findings and therapeutic strategies. Pharmacol Ther 2010; 128: 191-227.

17. Van Berlo JH, Maillet M, Molkentin JD. Signaling effectors underlying pathologic growth and remodeling of the heart. J Clin Invest 2013; 123 : $37-45$.

18. Brower GL, Gardner JD, Forman MF, et al. The relationship between myocardial extracellular matrix remodeling and ventricular function. Eur J Cardiothorac Surg 2006; 30: 604-610.

19. Hudlicka O, Brown M, Egginton S. Angiogenesis in skeletal and cardiac muscle. Physiol Rev 1992; 72: 369-417.

20. Taegtmeyer H, Sen S, Vela D. Return to the fetal gene program: a suggested metabolic link to gene expression in the heart. Ann N Y Acad Sci 2010; 1188: 191-198.

21. Lopaschuk GD, Jaswal JS. Energy metabolic phenotype of the cardiomyocyte during development, differentiation, and postnatal maturation. J Cardiovasc Pharmacol 2010; 56: 130-140.

22. Abel ED, Doenst T. Mitochondrial adaptations to physiological versus pathological cardiac hypertrophy. Cardiovasc Res 2011; 90: 234-242.

23. Schunkert H. Obesity and target organ damage: the heart. Int J Obes 2002; 26: 15-20.

24. Harmancey R, Wilson CR, Taegtmayer $H$. Adaptation and maladaptation of the heart in obesity. Hypertension 2008; 52: 181-187.

25. Gardin JM, McClelland R, Kitzman D, et al. Mmode echocardiographic predictors of six- to seven-year incidence of coronary heart disease, stroke, congestive heart failure and mortality in an elderly cohort (The Cardiovascular Health Study). Am J Cardiol 2001; 87: 1051-1057.

26. Vatner SF. Reduced subendocardial myocardial perfusion as one mechanism for congestive heart failure. Am J Cardiol 1988; 62: 94-98.

27. Dorn GW. Myocardial angiogenesis: its absence makes the growing heart founder. Cell Metab 2007; 5: 326-327.

28. Levine B, Yuan J. Autophagy in cell death: an innocent convict? J Clin Invest 2005; 115: 26792688.

29. Diwan A, Dorn GW. Decompensation of Cardiac Hypertrophy: Cellular Mechanisms and Novel Therapeutic Targets. Physiology 2007; 22: 56-64.

30. Narula J, Haider N, Virmani R, et al. Apoptosis in myocytes in endstage heart failure. $\mathrm{N}$ Engl J Med 1996; 335: 1182-1189. 
31. Liu Y, Cigola E, Cheng W, et al. Myocyte nuclear mitotic division and programmed myocyte cell death characterize the cardiac myopathy induced by rapid ventricular pacing in dogs. Lab Invest 1995; 73: 771-787.

32. Thiedeman KU, Holubarsch $\mathrm{CH}$, Medugorac I, Jacob R. Connective tissue content and myocardial stiffness in pressure overload hypertrophy: a combined study of morphologic, morphometric, biochemical, and mechanical parameters. Basic Res Cardiol 1983; 78: 140-155.

33. Scheuer J, Malhotra A, Hirsch C, Capasso J, Schaible TF. Physiologic cardiac hypertrophy corrects contractile protein abnormalities associated with pathologic hypertrophy in rats. J Clin Invest 1982; 70: 1300-1305.

34. Wankerl M, Schwartz K. Calcium transport proteins in nonfailing and failing heart: gene expression and function. J Mol Med 1995; 73: 487-496.
35. Neubauer S. The Failing Heart - An Engine Out of Fuel. N Engl J Med 2007; 356: 1140-1151.

36. Massoni F, Di Sabatino D, Ricci L, Onofri E, Ricci S. A rare case of myocardial infarction by myocardial bridging of circumflex artery. Clin Ter 2014; 165: 416-418.

37. Massoni F, Ricci S. Cardiac death by rupture of the right ventricular wall and hemopericardium. Cent Eur J Med 2014; 9: 45-48.

38. Massoni F, Ricci L, Di Sabatino D, Ricci S. Haemopericardium in hypercoagulability by diabetes and hypertension. Experim and Clin Card 2014; 20: 3100-3108.

39. Vitarelli A, Cortes Morichetti M, Capotosto L, et al. Utility of strain echocardiography at rest and after stress testing in arrhythmogenic right ventricular dysplasia. Am J Cardiol. 2013; 111: $1344-1350$ 